International Mathematical Forum, Vol. 8, 2013, no. 40, 1963 - 1975

HIKARI Ltd, www.m-hikari.com

http://dx.doi.org/10.12988/imf.2013.311217

\title{
More Around Pythagore from Ancient to Modern Times
}

\begin{abstract}
Albert Fässler ${ }^{1}$ and Xingjun Aries Tu ${ }^{2}$
Copyright (C) 2013 Albert Fässler and Xingjun Aries Tu. This is an open access article distributed under the Creative Commons Attribution License, which permits unrestricted use, distribution, and reproduction in any medium, provided the original work is properly cited.
\end{abstract}

\begin{abstract}
This work deals with the history around the "Pythagorean Theorem" and the "Pythagorean Number Triples" from 2200 B.C. until today. Special emphasis is done on china, including early applications. It also discusses the generalizations of the geometrical proof of $a^{2}+b^{2}=c^{2}$ : Any two polygons with the same area are equicomposable. But two polyhedrons with the same volume in general are not equicomposable. The latter was proven by Max Dehn as Hilbert's 3rd problem. The technique of Elliptic Curves allows to calculate different Rational Pythagorean Number Triples with equal area.
\end{abstract}

Mathematics Subject Classification: 11-03, 01A25, 11G05

Keywords: History, Pythagorean Theorem and Triples, Equicomposability in plane and space, Hilberts 3rd problem, Dudeney's famous example, Elliptic Curves to generate special Pythagorean Triples

\section{First Application in Ancient China ?!}

Chinese historical sources record that as early as in the 22nd century B.C., a person named Yu applied the so-called Pythagorean Theorem for water-control projects to prevent floods of the Yellow River. This was of great relevance for

\footnotetext{
${ }^{1}$ Fudan University, School of Mathematics, Shanghai 200433, under the Directory of ZongMin Wu and Berne University of Applied Sciences BFH-TI.

${ }^{2}$ Fudan University, Dept. of Chemistry, Shanghai and Dow Chemical Company, Shanghai.
} 
the people when planting crops for their living. This exciting information is mentioned in [12], where the two authors mainly cite the following Chinese historical records which still are accessible:

- Zhou Bi Suan Jing, finished by Sima Qian in the 1st century B.C.

- Shi Ji, written between 104 and 91 B.C. In English it means Historical Record. It describes individuals living in the long time span between the Huangdi monarch period (about 3000 B.C.) and part of the Han Dynasty, (about 200 B.C. to 220 A.C). Furthermore, about one hundred important personalities are portrayed. It also gives an outline of the different societies, as well as the situation of the minorities in as early as the late 22nd century B.C.

- Lu Shi Hou Ji Shi Er Zhu, written between 403 B.C. and 221 A.C. during the Warrier States Period.

Apparently, $\mathrm{Yu}$ was the first human being relevant to the theorem and applying it to solve a real world problem, long before Pythagore the Greek came into play! Yu obviously did so with great success, as is well known to almost everyone in China.

In a chapter of Shi Ji, called Shi Ji. Xia Ben Ji, the water-control project is described. It states that one needs tools to reach the goal, namely levelling instruments and two kinds of plot tools: compass for drawing circles and the carpenter's square to draw right angles. The Chinese characters describing these tools can be found in inscriptions of bones and on turtle shells of the Shang Dynasty (16th - 11th century B.C.) The chapter ends telling that the concept of the so-called Pythagorean theorem had penetrated into people's mind after $\mathrm{Yu}$ had successfully finished the water-control project. This may have been an important factor for $\mathrm{Yu}$ to be chosen as an emperor!

However, sinologists and historians have strong doubts about the existence of $\mathrm{Yu}$ : they consider this person as a mythological figure. The story about $\mathrm{Yu}$ cannot be found in either [13], a profound source for Chinese history)and its equivalent in French [15] nor in [2], [3], [4], [5], [7], [8], [9], [10] and [16].

This might explain the fact, that it will almost certainly never be possible to find out, if the mentioned statements about $\mathrm{Yu}$ is history or myth!

Remark: The story about Yu cannot be found in either [13], [15],[2], [3], $[4],[5],[7],[8],[9],[10]$ nor [16].

\section{Some Chinese History}

In many paintings of the Han dynasty, two of the earliest rulers hold an instrument with two legs similar to a compass and a right angle ruler like a carpenter's square in their hands. 
At the beginning of the first chapter of the book Zhou Bi Suan Jing which is older than the Jiu Zhang Suan Shu or Nine Chapters on the Mathematical Art (written during the Han-period, about 200 B.C. to 220 A.C.), the following is recorded: In the 11th century B.C., during the Zhou dynasty in the west of today's China, a conversation between the Monarch and a man called Shang Gao put forward the theorem. Shan Gao made a statement in the following sense: If the lengths of the two line segments forming a right triangle are 3 and 4 , then the hypotenuse of the triangle has length 5. Furthermore, the relation $c=\sqrt{a^{2}+b^{2}}$ is clearly recorded in ancient China. This relationship is known as Gou San Gu Si Xian Wu to every schoolchild in China.

In Zhou Bi Suan Jing we also find that at that time it was known to use the similarity of right triangles to calculate distances. Shang Gao gave this information: In placing the carpenter's square appropriately, one can measure the height, the depth and the distance by use of similarity.

Zhou Bi Suan Jing also records a dialog that took place in the 6th or 7th century B.C. between the two people Rong Fang and Mr. Chen. They discussed a method to calculate the height $\mathrm{H}$ of the sun (see Figure 1).

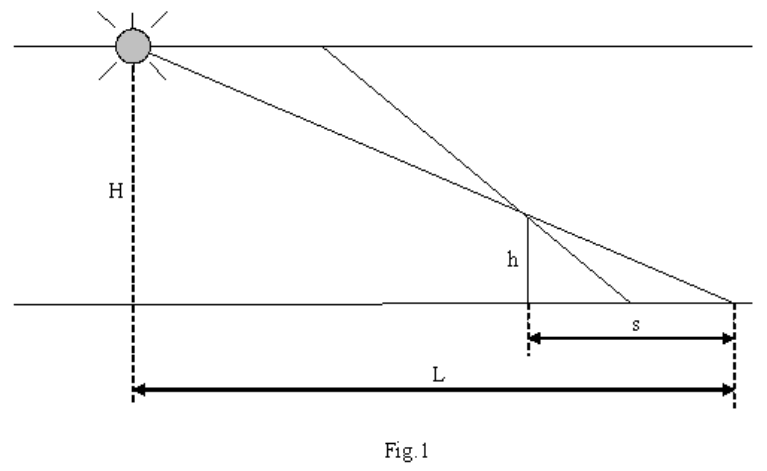

Figure 1: Height of the sun

In contemporary English it would go as follows: At the summer solstice, a pole of length $\mathrm{h}=8 \mathrm{Chi}$ long $(1 \mathrm{Chi}=1 / 3 \mathrm{~m})$ casts a shadow of length $\mathrm{s}=1 \mathrm{Chi}$ and $3 \mathrm{Cun}(10 \mathrm{Cun}=1 \mathrm{Chi})$. If the shadow changes by $1 \mathrm{Cun}$, then the sun moves $1000 \mathrm{Li}(1 \mathrm{Li}=1 / 2 \mathrm{~km})$ towards the south. By the similitude principle (1 Chun : $1000 \mathrm{Li}=\mathrm{h}: \mathrm{H}$ ), they obtained the sun's height $\mathrm{H}=80000 \mathrm{Li}$.

In their opinion the shadow disappears, when the sun has moved about $\mathrm{L}=60$ '000 Li to the south. They calculated the original distance between the sun and the pole (see Figure 2) to be $\sqrt{8^{2}+6^{2}} \times 10^{4}=10 \times 10^{4} \mathrm{Li}$.

The author's explanation is: To obtain the distance, just multiply by itself (obviously describing the square), and gives further comments on the square root. Of course, this calculation is not correct from the astronomical point of view. 


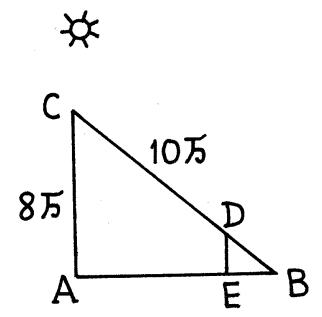

Figure 2: Distance of the sun

Remark: The units Li, Chi, Cun are still in use today, but their measure might have changed since.

The cases mentioned show that even real world applications of the algebraic aspect of the theorem $c^{2}=a^{2}+b^{2}$ were performed very early in Ancient China, almost in contradiction to some comments and assumptions of van der Waerden in his book [10]:

How can we explain the fact that methods for finding Pythagorean triples found their way into Babylonian, Hindu, Greek and Chinese texts? For what purposes were these methods invented and transmitted to later generations? The clearest exposition of these methods is found in the Chinese Nine Chapters. So it is only natural to ask: For what purpose were these triples used in the Nine Chapters? [...] Answer: The final chapter of this treatise consists of a sequence of problems with solutions, all concerning right triangles. The Chinese collection of problems is closely related to Babylonian collections. The similarity is so close that a Pre-Babylonian common origin must be assumed [...] I now suppose that Pythagorean triples were invented just for this purpose: to compose problems with rational solutions.

Perhaps the most influential of all Chinese mathematical books was the before-mentioned Nine Chapters on the Mathematical Art. Its last chapter includes problems on right triangles, some of which later reappeared in India and Europe. A well-known problem is that of "the broken bamboo":

A bamboo 10 Chi high, the upper end of which, being broken, reaches the ground 3 Chi from the stem. Find the height of the break. The book also includes problems on surveying, agriculture, engineering, taxation, calculation, solving equations, and on the properties of the right triangles.

The so called "Chinese rule" or "Chinese method" for calculating Pythagorean triples can be written as follows:

$$
a=\alpha \beta \quad b=\left(\alpha^{2}-\beta^{2}\right) / 2 \quad c=\left(\alpha^{2}+\beta^{2}\right) / 2
$$

Therefore, this parametrization for all Pythagorean number triples is obviously much older than Fermat. In addition to that, in Chou Pei Suan Ching (see [16], Chapter 19), written between 3rd century B.C. and 3rd century A.C., 
we find a proof of the Pythagorean Theorem for the case 3, 4 and 5. But the idea is general in nature (see Figure 3).

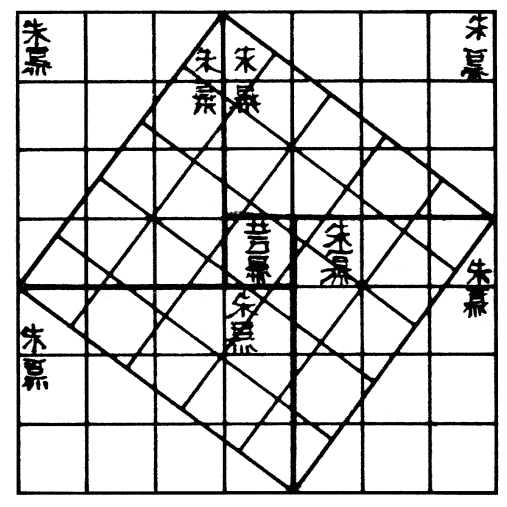

Figure 3: Proof in the Chou Pei Suan Ching

In Zhou Bi Suan Jing, we also find a clear description of $c=\sqrt{a^{2}+b^{2}}$, including the square root and the statement that the vertices of all right triangles over a common fixed hypotenuse lie on a circle.

\section{More History of different Civilizations}

The Sulvasutras in India are ancient Hindu manuals which were probably written between 500 B.C. and 200 A.C. They embody geometric rules for the construction of altars by rope stretching and show an acquaintance with Pythagorean triples. It contains some geometrical constructions, but also calculations using the identity $(c-b)(c+b)=a^{2}$. Some of the geometrical constructions are already mentioned in the earlier Satapatha Brahmana which was written between 1000 and 800 B.C.

Long before the Sulvasutras, the Babylonians (1700 B.-100 B.C.) have computed Pythagorean triples. The degree of influence of Greek, Babylonian, and Chinese mathematics on Hindu mathematics, and vice versa, is still an unsettled matter, but there is ample evidence that there was considerable influence in both directions. From a very early date on, India exchanged diplomats with both the West and the Far East.

The precise orientation of the foundations of the immense structures of ancient Egypt with the four cardinal points of the compass led some historians to surmise that these "rope stretchers" were able to construct right angles using ropes divided by two knots into sections that were in the proportion of $3: 4: 5$. However simple this approach may appear today, no surviving document from antiquity confirms that it actually took place. It is often said 
that the ancient Egyptians were familiar with the Pythagorean theorem, but there is no hint on the papyri that have come down to us. It does not appear in any form in surviving documents from Egypt, but tablets even from the Old Babylonian Period show that in Mesopotamia the theorem was widely used.

We do not know, if megalithic man knew the theorem. Perhaps not, but he was feeling his way towards it. One can almost say that he was obsessed by the desire to discover and record in stone as many triangles as possible that were right triangles and yet had all three sides of integral lengths.

Long before Diophantus (about 250 A.C.), the Babylonians knew how to calculate Pythagorean triples: Plimpton 322, the famous stone stab with several triples on it, was created under the dynasty of Hammurabi.

Fibonacci (also called Leonardo of Pisa, 1175-1250) proved that all Pythagorean triples can be generated by the parametrisation (1).

Van der Waerden wrote in [10]: We have seen so many similarities between the mathematical and religious ideas current in England in the Neolithic Age, in Greece, in India, and in China in the Han-period that we are bound to postulate the existence of a common mathematical doctrine from which these ideas were derived. [...]

The general rule for computing Pythagorean triangles is found only in the very late treatise of Diophantus. So the only reasonable explanation of the many similarities seems to be, once more, the hypothesis of a common origin.

The question arises: Is it possible that Chinese Algebra was derived from Babylonian Algebra? For several reasons, I don't think that such a derivation is possible. First, Chinese Algebra is on a higher mathematical level. [...] Secondly, the Chinese text is richer in geometrical notations and methods. The knowledge of the Theorem of Pythagoras does not necessarily imply the calculation of Pythagorean triangles. [...]

\section{Ancient Chinese Proof by Decomposition and Hilbert's 3rd Problem}

It seems that proofs of the theorem occurred much later than its applications. Zhou Bi Suan Jing did not give any. It was in the 3rd century A.C., when in China, in the time period of the Three Kingdoms, Zhao Shuang gave a strict and ingenious proof of it.

There are a variety of proofs, based on different dissections. It is interesting to notice that the decomposition problem was posed by David Hilbert (18621943) at the International Congress of Mathematicians in 1900 in a general setting. 
In the same year, before Hilbert's talk in Paris, Max Dehn (1878-1952) gave the solution, showing that solid figures of equal volume are not necessarily equicomposable by dissection into a finite number of polygons. Certain tetrahedrons cannot be dissected into a cube such as for example the regular one. His solution is a purely algebraic one (by the so called Dehn invariant) for a geometrical problem (see [1]). Two polyhedrons are said to be equicomposable, if one polyhedron can be suitably decomposed (dissected) into a finite number of polyhedrons in a way such that it can be reassembled to give the other polyhedron. Historically, Dehn was the first to solve a Hilbert problem.

Interestingly enough, the analog question in the plane with polygons was answered positively much earlier. F.Bolyai (the father of the famous J.Bolyia who developed ideas of non-Euclidian geometry) in 1832 and the German officer and amateur mathematician P. Gerwien in 1833 independently of each other proved that two polygons have the same area if and only if they are equicomposable.

An outstanding example of a dissection of an equilateral triangle into four pieces so as to reassemble into a square was given by Henry Ernest Dudeney (1857-1930):

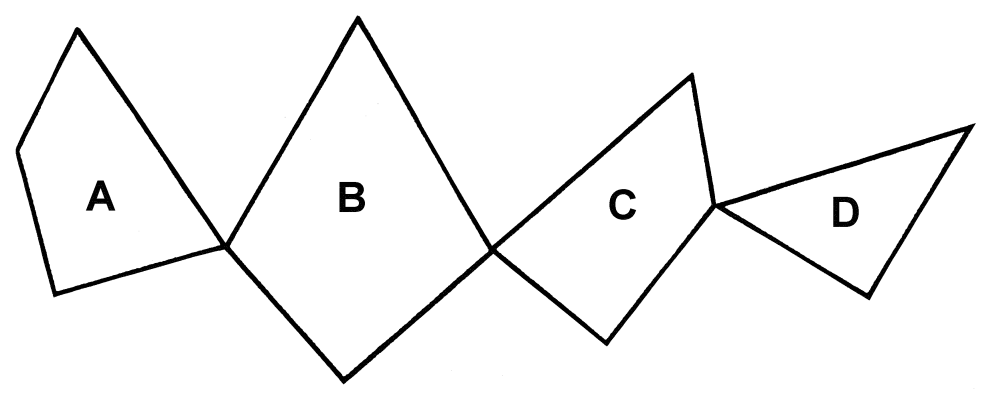

Figure 4: Four hinged polygons 
A remarkable feature of this puzzle is the fact that the pieces can be hinged at three vertices to form a chain (see Figure 4) that can be closed clockwise to get the triangle and counter-clockwise to get the square (see Figure 5). Dudeney demonstrated this fact with a hinged mahagony model at the Royal Society in 1905.

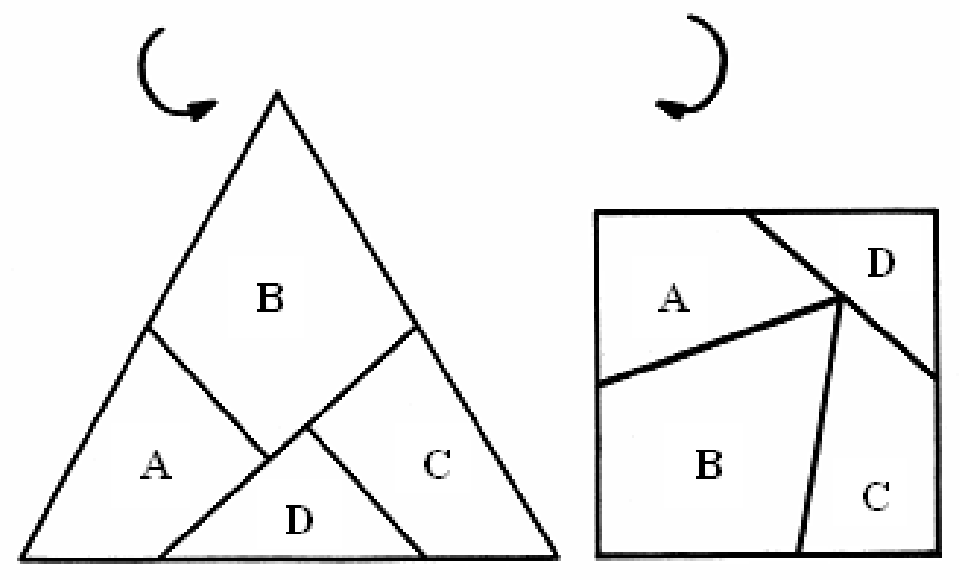

Figure 5: Triangle and square

In 1948, the Swiss mathematicians H. Hadwiger and P. Glur gave necessary and sufficient conditions for two polygons of the same area to be equidecomposable, allowing solely translations of the different pieces (see [1]).

\section{$5 \quad$ Pythagorean Triples and Elliptic Curves}

It is well known that Elliptic Curves play an important role in modern Cryptology [11]. Interesting enough, there is a link between rational Pythagorean tripels with a certain common integer area $n$ of their corresponding triangles and a specific Elliptic curve:

Starting off with

$$
\begin{gathered}
a^{2}+b^{2}=c^{2} \\
a \cdot b=2 n
\end{gathered}
$$

leads to

$$
\begin{aligned}
& \left(\frac{a+b}{2}\right)^{2}=\frac{a^{2}+b^{2}+2 a b}{4}=\frac{c^{2}+4 n}{4}=\left(\frac{c}{2}\right)^{2}+n \\
& \left(\frac{a-b}{2}\right)^{2}=\frac{a^{2}+b^{2}-2 a b}{4}=\frac{c^{2}-4 n}{4}=\left(\frac{c}{2}\right)^{2}-n
\end{aligned}
$$


Therefore, the $x=\left(\frac{c}{2}\right)^{2}$ has to be a square of a rational number as well as $x+n$ and $x-n$. This means that we have to search for triples

$$
x-n, x, x+n
$$

in arithmetic progression with step $n$, such that they are all squares of rational numbers. It follows that $(x-n) x(x+n)=x^{3}-n^{2} \cdot x$ has to be a square of a rational number as well.

In other words: It is necessary that we can find a rational solution of the Elliptic curve

$$
y^{2}=x^{3}-n^{2} \cdot x
$$

But such a solution does not imply that $x+n$ and $x-n$ are also squares of a rational numbers. Example:

$$
n=5, \quad(x, y)=\left(\frac{25}{4}, \frac{75}{8}\right) \quad \text { which gives } \quad x+5=\frac{45}{4}
$$

However, if we had a rational point $P\left(x_{0}, y_{0}\right)$ with $y_{0} \neq 0$ on (4), the intersection of the tangent line at $P$ with the Elliptic curve gives an $\mathrm{x}$-value, noted $x_{1}$, such that all three values $x_{1}-n, x_{1}, x_{1}+n$ are squares of rational numbers which can be seen as follows:

Implicit differentiation of (4) yields to $2 y y^{\prime}=3 x^{2}-n^{2}$ and we get the slope

$$
y^{\prime}\left(x_{0}\right)=\frac{3 x_{0}^{2}-n^{2}}{2 y_{0}}
$$

For the tangent line, we have

$$
y(x)=y^{\prime}\left(x_{0}\right) \cdot x+\left[y_{0}-y^{\prime}\left(x_{0}\right) \cdot x_{0}\right]
$$

And the intersection now gives

$$
\left[y^{\prime}\left(x_{0}\right) \cdot x+c\right]^{2}=x^{3}-n^{2} x
$$

It follows

$$
p(x)=x^{3}-y^{\prime}\left(x_{0}\right)^{2} x^{2}+\text { rest }=0
$$

Since this cubic equation has the double root $x_{0}$, we have on the other side

$$
p(x)=\left(x-x_{0}\right)\left(x-x_{0}\right)\left(x-x_{1}\right)=x^{3}-\left(x_{0}+x_{0}+x_{1}\right) x^{2}+\text { rest }=0
$$

Comparance of the second coefficient gives $2 x_{0}+x_{1}=y^{\prime}\left(x_{0}\right)^{2}$. Therefore

$$
x_{1}=y^{\prime}\left(x_{0}\right)^{2}-2 x_{0}=\frac{\left(3 x_{0}^{2}-n^{2}\right)^{2}-8 x_{0} y_{0}^{2}}{4 y_{0}^{2}}
$$


Using $y_{0}^{2}=x_{0}^{3}-n^{2} x_{0}$ in the numerator supplies

$$
x_{1}=\frac{9 x_{0}^{4}-6 x_{0}^{2} n^{2}+n^{4}-8 x_{0}^{4}+8 n^{2} x_{0}^{2}}{4 y_{0}^{2}}=\frac{x_{0}^{4}+2 n^{2} x_{0}^{2}+n^{4}}{4 y_{0}^{2}}=\left(\frac{x_{0}^{2}+n^{2}}{2 y_{0}}\right)^{2}
$$

But now, we have also

$$
\begin{aligned}
& x_{1}+n=\frac{\left(x_{0}^{2}+n^{2}\right)^{2}+4 n\left(x_{0}^{3}-n^{2} x_{0}\right)}{4 y_{0}^{2}}=\frac{x_{0}^{4}+n^{4}+2 x_{0}^{2} n^{2}+4 n x_{0}^{3}-4 n^{3} x_{0}}{4 y_{0}^{2}} \\
& x_{1}+n=\frac{x_{0}^{4}+n^{4}+\left(4 x_{0}^{2} n^{2}-2 x_{0}^{2} n^{2}\right)+4 n x_{0}^{3}-4 n^{3} x_{0}}{4 y_{0}^{2}}=\left(\frac{x_{0}^{2}-n^{2}+2 x_{0} n}{2 y_{0}}\right)^{2}
\end{aligned}
$$

(see the three leading squares) and similar

$$
x_{1}-n=\frac{x_{0}^{4}+n^{4}+\left(4 x_{0}^{2} n^{2}-2 x_{0}^{2} n^{2}\right)-4 n x_{0}^{3}+4 n^{3} x_{0}}{4 y_{0}^{2}}=\left(\frac{-x_{0}^{2}+n^{2}+2 x_{0} n}{2 y_{0}}\right)^{2}
$$

This procedure can be repeated with a tangent line at the point $\left(x_{1}, y_{1}\right)$ etc. to get an infinity of rational Pythagorean triples which correspond to triangles with a common area $n$.

We want to calculate the corresponding Pythagorean triple to $x_{1}$ now:

$x_{1}=(c / 2)^{2}$ implies $c=2 \sqrt{x_{1}}$.

The tangent line equation (6) delivers

$$
y_{1}=y\left(x_{1}\right)
$$

On the other side, using (2)and (3) we have also

$$
y_{1}=\sqrt{(x-n) x(x+n)}=\frac{(a-b) \cdot c \cdot(a+b)}{8}=\frac{c\left(a^{2}-b^{2}\right)}{8}
$$

Therefore we know

$$
a^{2}-b^{2}=\frac{8 y_{1}}{c}
$$

as well as

$$
a^{2}+b^{2}=c^{2}
$$

Addition and subtraction of the last two equations delivers $a^{2}, b^{2}$ reps. $a, b$. It is a delicate question to ask which integers $n$ are possible areas of ppt's.

A necessary condition (but by far not sufficient) for $n$ is the fact that it is square free (which means: each prime facor of $n$ appears once only). For example 5 and 21 are OK, but not 9 and 45. See the Conjecture of BirchSwinnerton-Dyer. 


\section{A numerical example:}

We start from the pythagorean triple $(a, b, c)=(35,12,37)$ with area $n=210$ and calculate the corresponding point $\left(x_{0}, y_{0}\right)$ on the Elliptic curve

$$
y^{2}=x^{3}-44100 x
$$

The value $x_{0}=(37 / 2)^{2}=1369 / 4$ substituted in (10) gives

$$
y_{0}^{2}=1599760009 / 64 \Longrightarrow y_{0}=39997 / 8 \text {. }
$$

The slope (5) is

$$
m=y^{\prime}\left(x_{0}\right)=4916883 / 159988
$$

Now we can calculate

$$
x_{1}=m-2 x_{0}=\frac{6655166817121}{25596160144}
$$

substituted into the tangent line (6) gives

$$
y_{1}=m \cdot x_{1}+\left(y_{0}-m x_{0}\right)=\frac{10123232905622052719}{4095078469118272}
$$

From our new point $\left(x_{1}, y_{1}\right)$ on the Elliptic curve, we construct the corresponding pyhthagorean number triple:

$$
c=2 \sqrt{x_{1}}=\frac{2579761}{79994}
$$

Therefore, the right hand side of $(8)$ is

$d=\frac{8 y_{1}}{c}=3924097195679 / 6399040036$

and the right hand side of $(9)$ is

$s=c^{2}=6655166817121 / 6399040036$.

Finally we have

$$
a=\sqrt{\frac{s+d}{2}}=\frac{31080}{1081} \quad b=\sqrt{\frac{s-d}{2}}=\frac{1081}{74}
$$

Check: It is indeed

$$
\frac{a b}{2}=210 \text { and } a^{2}+b^{2}=c^{2}
$$

As has been shown, with this process we could generate an infinity of rational triangles with common area 210. Interesting enough, there are two ppt's triples with integer values: $(21,20,29)$ and $(35,12,37)$.

Already Fermat gave a method to generate an arbitrary number of right triangles with rational sides and suitably chosen common area ( Dickson: History of Theory of Numbers, Vol.2). 
Questions about different primitive Pythagorean triples with equal areas $\frac{1}{2} x y$ are open. With his C-program, Max Hailperin (St. Olafs College in Minneapolis) found 5 different areas, which allow 3 different primitive Pythagorean triples each.

Using Euclids parametrisation $a=\alpha^{2}-\beta^{2}, \quad b=2 \alpha \beta, \quad c=\alpha^{2}+\beta^{2}$ with $\operatorname{gcd}(\alpha, \beta)=1$ and $\alpha+\beta=1(\bmod 2)$ and $\alpha>\beta$

for $a^{2}+b^{2}=c^{2}$ we get the area

$$
n=\alpha \beta\left(\alpha^{2}-\beta^{2}\right)
$$

Here are the parameters $(\alpha, \beta)$ for the 5 cases with its square-free areas $n$ :

1. $(77,38) ;(78,55) ;(138,5)$

$$
n=13123110=2 \cdot 3 \cdot 5 \cdot 7 \cdot 11 \cdot 13 \cdot 19 \cdot 23
$$

2. $(3422,55) ;(3306,61) ;(2035,266)$

$$
n=2203385574390=2 \cdot 3 \cdot 5 \cdot 7 \cdot 11 \cdot 13 \cdot 19 \cdot 29 \cdot 37 \cdot 59 \cdot 61
$$

3. $(2002,1817) ;(2622,143) ;(1610,869)$

$$
n=2570042985510=2 \cdot 3 \cdot 5 \cdot 7 \cdot 11 \cdot 13 \cdot 19 \cdot 23 \cdot 37 \cdot 67 \cdot 79
$$

4. $(2201,1166) ;(3565,198) ;(2438,2035)$

$$
n=8943387723270=2 \cdot 3 \cdot 5 \cdot 7 \cdot 11 \cdot 13 \cdot 23 \cdot 31 \cdot 37 \cdot 53 \cdot 71
$$

5. $(9077,1122) ;(10434) ;(7238,2465)$

$$
n=826290896699730=2 \cdot 3 \cdot 5 \cdot 7 \cdot 11 \cdot 17 \cdot 29 \cdot 31 \cdot 37 \cdot 43 \cdot 47 \cdot 313
$$

Euler (1707-1783) knew the first case.

\section{References}

[1] V. G. Boltianskii (translated by R. A. Silverman), Hilbert's Third Problem, Wiley, New York, (1978).

[2] C. B. Boyer, A History of Mathematics, Wiley, New York, 2nd ed. (1989).

[3] D. M. Burton, The History of Mathematics, An Introduction, Allyn and Bacon Inc., (1985).

[4] H. Eves, An Introduction to the History of Mathematics, Saunders College Publishing, 5th ed., (1983).

[5] A. Reader, ed. J. Fauvel and J. Gray, The History of Mathematics, Macmillan Education Ltd., London, (1987). 
[6] A. Fässler, Multiple Pythagorean Number Triples, American Mathematical Monthly (1991), 505-517.

[7] R. Hartshorne, Companion to Euclid, A course of geometry, based on Euclid's Elements and its modern descendants, Lecture Notes, Vol.5, American Mathematical Society, (1991).

[8] O. Neugebauer, Vorlesungen über Geschichte der antiken mathematische Wissenschaften, Erster Band: Vorgriechische Mathematik,Springer, 2nd ed., (1969).

[9] H. Selin and U. D'Ambrosio, Mathematics Across Cultures, The History of Non-Western Mathematics, Kluwer Academic Publisher, 2000, transferred to digital print (2001).

[10] B. L. van der Waerden, Geometry and Algebra in Ancient Civilizations, Springer, 2nd ed., (1983).

[11] L. C. Washington, Elliptic Curves, Number Theory and Cryptography, Series Discrete Mathematics and Its Applications, Chapman \& Hall, (2003).

[12] Jingan Chen and Yingcheng Sun, Zhongwai Shuxue Jianshi, Chinese Mining Industry University Xuzhou, Jiangsu Province, (2002).

[13] Sima Qian, Records of the Grand Historian of China, Translation by Burton Watson from the Shih chi of Ssu-ma Ch'ien, Columbia University Press, Vol.I (1961).

[14] L. E. Dickson, History of the Theory of Numbers, Volume II, Chelsea Publishing Company, New York, (1971).

[15] Sima Qian, Les mémoires historiques de Ssu-ma Ch’ien Vol.I, Traduction par Edouard Chavannes, Ernest Leroux, Paris, (1895-1905).

[16] J. Needham, Science and Civilisation in China, Volume 3, with collaboration of Wang Ling, Cambrige University Press, (1959).

\section{Received: November 17, 2013}

